\title{
Review of the Biomolecular Modification of the Metal-Organ-Framework
}

\author{
Qiqi Xing, Yixiao Pan, Yihe Hu and Long Wang* \\ Department of Orthopedics, Xiangya Hospital, Central South University, Changsha, China
}

Metal-organ frameworks (MOFs), as a kind of novel artificial material, have been widely studied in the field of chemistry. MOFs are capable of high loading capacities, controlled release, plasticity, and biosafety because of their porous structure and have been gradually functionalized as a drug carrier. Recently, a completely new strategy of combining biomolecules, such as oligonucleotides, polypeptides, and nucleic acids, with MOF nanoparticles was proposed. The synthetic bio-MOFs conferred strong protection and endowed the MOFs with particular biological functions. Biomolecular modification of MOFs to form bridges for communication between different subjects has received

OPEN ACCESS

Edited by:

Feng Luo,

East China University of

Technology, China

Reviewed by:

Peng Zhou,

University of Electronic Science and Technology of China, China Han Wen Cheng,

Shanghai Institute of Technology, China

*Correspondence: Long Wang

dr_wanglong@csu.edu.cn

Specialty section:

This article was submitted to Inorganic Chemistry

a section of the journal

Frontiers in Chemistry

Received: 14 April 2020

Accepted: 22 June 2020

Published: 28 July 2020

Citation:

Xing Q, Pan Y, Hu Y and Wang L (2020) Review of the Biomolecular Modification of the

Metal-Organ-Framework

Front. Chem. 8:642.

doi: 10.3389/fchem.2020.00642 increased attention. This review will focus on bio-MOFs modification methods and discuss the advantages, applications, prospects, and challenges of using MOFs in the field of biomolecule delivery.

Keywords: MOF, biomolecular, modification, delivery, nano material

\section{BACKGROUND}

The metal-organ framework (MOF), which is also known as a porous coordination polymer (PCP), is a kind of novel nanomaterial composed of metals or metal clusters, chains or layers formed by non-toxic metals ( $\mathrm{Fe}, \mathrm{Zn}, \mathrm{Ca}, \mathrm{Mg}$, etc.) and organic compounds, such as carboxylic acid and phosphonic acid (Férey, 2008). PCPs have been widely used in the fields of chemistry and material science in applications such as gas separation and storage (Eddaoudi et al., 2002), sensing (Xu et al., 2011), catalysis (Zhuang et al., 2015), and chromatography (Li et al., 2009; Xiao et al., 2009). MOFs have a porous structure with pore sizes between 0.4 and $6 \mathrm{~nm}$ (Zhou et al., 2012). The inherent pore size and structure can be modulated according to the organic-metal compound category and the spatial arrangement. These characteristics enable MOFs to possess open architectures that allow them to combine with, penetrate, and encapsulate a variety of molecules. Therefore, MOFs are ideal materials for storage, protection (Liang et al., 2016b) and carrier functions (Morris and Wheatley, 2008). Recently, non-toxic and biocompatible MOFs, as newly developed materials, have been recommended for use in biological field applications, such as bio-imaging, drug delivery, and electrocatalysis (Huxford et al., 2010; Cai et al., 2015), and these functions were primarily achieved by incorporation of drugs or biological molecules with MOFs (Wang et al., 2015). Increasing numbers of drugs have been combined with different kinds of MOFs, including isoniazid (INH), which is a traditional antitubercular agent that has been loaded onto Fe-MIL-101- $\mathrm{NH}_{2}$ nanoparticles to control the release of the drug and improve absorption by macrophages (Wyszogrodzka-Gaweł et al., 2019). An antibiotic (vancomycin) and targeting ligand (folic acid) could also be combined with zeolitic imidazolate framework (ZIF-8) nano MOFs to be delivered to Staphylococcus aureus to control infection (Chowdhuri et al., 2017). Furthermore, 
loading with antitumoral and retroviral drugs to fight cancer and AIDS has greatly inspired MOF research (Horcajada et al., 2009). These successes have promoted the development of MOFs for use in the field of biology.

Biomolecules are special organic molecules produced by living systems, including nucleic acids, proteins, polysaccharides, and lipids, which cannot easily pass through a cell membrane due to their large weight and volume. Biomolecules can also be present on the cell membrane alone, but this occurs with difficulty (Whitehead et al., 2009) owing to the effect of serum nuclease (Keles et al., 2016). Delivery systems have emerged to offer superior thermal and chemical protection for biomacromolecular cargo, which is mainly divided into virus and non-viral vectors (Yoo et al., 2011; Kotterman et al., 2015). Compared with non-viral vectors, the low selectivity, potentially unsafe nature, and insertion mutations (Thomas et al., 2003; Waehler et al., 2007; Mintzer and Simanek, 2009) of viruses have limited their application in living bodies. Non-viral vectors, including organic carriers (liposomes, polymers, peptides, etc.) and inorganic carriers (silica, carbon tubes, calcium phosphate, etc.) (Sokolova and Epple, 2008; Nam et al., 2009), are also considered to be defective (Yin et al., 2014; Chira et al., 2015). Among them, poly-L-lysine, which is a cationic polymer, is one of the most common polymers used for DNA delivery (Khalil et al., 2006; Cheng and Lee, 2016). However, its biotoxicity, as well as its ATPase reduction, autophagy (Lv et al., 2006) and immunogenic responses, cannot be ignored (Tseng et al., 2009; Wan et al., 2013). Additionally, as the only non-viral delivery systems currently in clinical trials, lipid microsystems (Ginn et al., 2018) have been suggested to form large aggregates that hinder the further transportation of nanoparticles in blood (Dash et al., 1999; Das et al., 2015). It is necessary to develop a new biomacromolecule carrier with good biocompatibility and biodegradability.

As porous nanomaterials, MOFs are capable of high loading capacities, controlled release, plasticity, and biosafety, which facilitate the selective transportation of nanomaterials through the porous network (An et al., 2012). MOFs have been proposed as an attractive alternative to mitigate drawbacks that other drug delivery systems (DDSs) face (Abanades Lazaro et al., 2020). Thus, MOFs have already served as a successful drug delivery platform (Zhao et al., 2011). However, there are many differences between drug and biomolecule delivery systems (Zhuang et al., 2017). First, the electrostatic interactions and covalent bonds used to incorporate drugs might not be suitable for the incorporation of biomolecules and MOFs. Second, MOF stability should be a consideration because the addition of biomolecules might change the crystal structure. Third, targeting will be expected. Last, but not the least, a controlled-release effect is another characteristic to be encouraged. Among these requirements, the most important is the effective protection of biomolecules from external factors. In addition, the simplicity and reproducibility of material manufacturing should also be taken into account (Morris and Wheatley, 2008; Imaz et al., 2011). At present, there are two ways to combine MOFs with biomolecules. One method involves growing or depositing metal ions and organics onto a two-dimensional plane to form MOFs films, which are used to physically wrap the target molecule
(Horcajada et al., 2011; Shekhah et al., 2011). The other method involves chemical bonding, including surface modification and internal encapsulation (Doonan et al., 2017), which is particularly useful due to its high stability, capacity, and affinity.

This review will focus on the reported methods of bioMOFs functional synthesis, including surface modification and internal encapsulation, and further discuss the advantages, applications, prospects, and challenges of MOFs in the field of biomolecule delivery.

\section{MOF BIOFUNCTIONALIZATION BY SURFACE MODIFICATION}

In pioneering research, the biochemical surface modification of nanomaterials has been applied to biological probes and cell imaging (Wang D. et al., 2019). To date, MOFs have been modified on their surface to meet specific requirements and achieve biological functionalization, such as targeted delivery and localized release (Meng et al., 2018). These characteristics greatly improve the performance and bioutilization of MOFs (Cai et al., 2017; Huang et al., 2018). Consequently, there are two generalizable methods that postsynthetically functionalize the bulk MOF structure with biomolecules (Wang et al., 2015). The first method is the covalent combination of the modifier with an anchor on the surface of prepared MOFs before MOF synthesis. Unlike the modification of a pre-synthesized organic linker, the second method involves the coordination of the modifier directly on the surface of the post-synthesized MOF. The chelation of metal ions with target molecules is responsible for the connection (Deria et al., 2015). The unsaturated coordination metal sites on MOFs allow for the incorporation of biomolecules, and UiO-66 will be used to discern the two methods (Figure 1).

Via the first method, the conjugation of nucleic acid and azidefunctionalized UiO-66- $\mathrm{N}_{3}\left(\mathrm{Zr}_{6} \mathrm{O}_{4} \mathrm{OH}_{4}\left(\mathrm{C}_{8} \mathrm{H}_{3} \mathrm{O}_{4}-\mathrm{N}_{3}\right)\right)$, which is transferred from UiO-66, was produced (Morris et al., 2014). Alkane terminal ligands could react with the azide group in UiO-66- $\mathrm{N}_{3}$ via click reactions, which have been utilized to interface MOFs in bulk with a variety of organic functionalities. By the click reaction, $\mathrm{UiO}-66-\mathrm{N}_{3}$ was able to interact with dibenzyl-cyclooctyne (DBCO)-functionalized DNA and further realize cellular entry. Furthermore, the functionalized MOFs gained the ability to hybridize with diverse complementary nucleic acids in a sequence-specific fashion, which provided the possibility of the widespread bioapplication of this kind of MOF. However, the degree of DNA surface combination obtained by the first approach was approximately two times lower than that achieved by the other method, in which the surface modification of oligonucleotides resulted from metal-phosphate coordination through modified DNA and unsaturated metal sites on the MOF surface (Wang S. et al., 2017). In that process, chemically modified phosphonamidites at either the $3^{\prime}$ or $5^{\prime}$ end of the oligonucleotide were added into the colloidal suspension of synthesized UiO-66, and then the terminal phosphate moiety was adsorbed onto the MOF nanoparticles by coordination with the solvent-bound $\mathrm{Zr}$ sites (Deria et al., 2015). The affinity of the terminal 


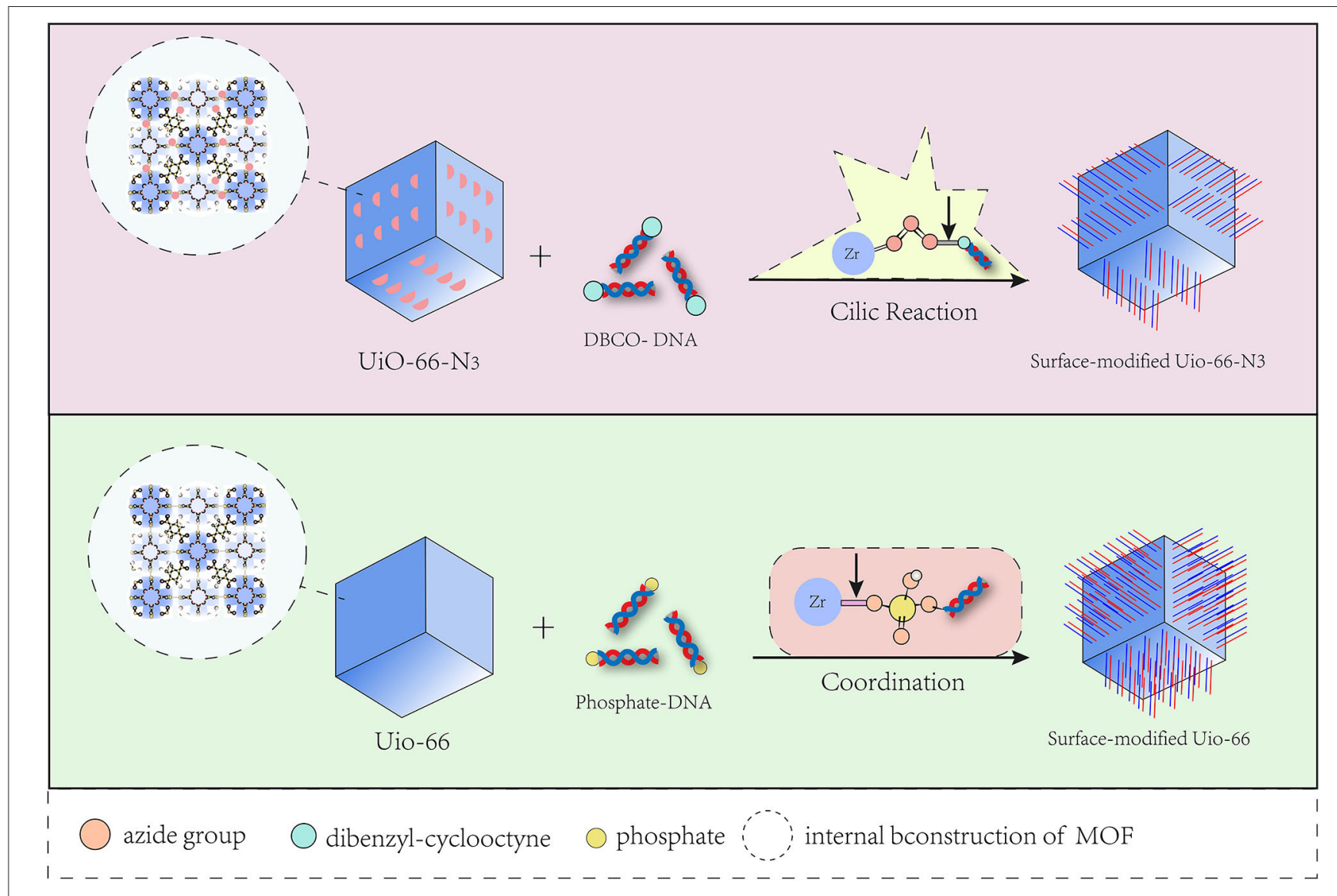

FIGURE 1 | Two methods for surface modification to MOF by DNA.

phosphate for the $\mathrm{Zr}$ centers was better than that of the internal phosphodiester bonds of oligonucleotides because of the increased steric hindrance encountered by the internal phosphodiester. In addition, the DNA surface coverage directly correlated with the surface second building unit (SBU) density, metal-phosphate coordination number, and bond strength of the MOF. Therefore, increased SBU coordination numbers yield higher DNA functionalization densities. Additionally, it should be mentioned that this approach to DNA-modified MOFs was independent of the category of organic linkers and was broadly applicable to a variety of metal clusters. This means that it is a general way to synthesize the regular structure of surfacemodified DNA-MOFs. Based on this method, a research team then designed a nucleic acid-MOF nanoparticle using NU-1000 and PCN-222/MOF-545, which acted as a host to easily and effectively deliver a variety of proteins into cells (Wang S. et al., 2019).

Through in-depth research, many kinds of biomolecules have been developed for the surface modification with different MOFs, such as oligopeptides that form metalpeptide frameworks with copper and calcium due to carboxylic acid chelation (Mantion et al., 2008). Surface combination has become an important and general way to obtain biofunctionalized MOFs.

\section{MOF BIOFUNCTIONALIZATION BY INTERNAL MODIFICATION}

Unlike surface modification, internal modification involves a higher-level combination, which involves the encapsulation of molecules by MOFs. The molecules can be better protected and bound more tightly by internal modification than by surface modification. Through encapsulation, MOFs could act as carriers to deliver large drugs and biomolecules, as well as to achieve the condition-triggered release of drugs from a vehicle (Roth et al., 2018). Compared with drugs, proteins, and nucleotides have more complicated multistructures, which means that conformational changes are always unavoidable and might disrupt their function (Wickner, 2005) when they are transported through nano channels to enter the cell. Therefore, a carrier would be necessary and important. Among nano delivery systems, MOFs are novel and outstanding. Taking enzymes as an example, MOF (Hudson et al., 2008) crystals have an increased encapsulation efficiency, recyclability, and an excellent enzyme-catalytic performance (Rapoport, 2007) compared to mesoporous silica (Lykourinou et al., 2011). However, biomacromolecules similar in size to MOF pores were hardly able to be loaded into the framework via postsynthetic infiltration (Chen et al., 2012). Recently, two methods for 
encapsulating target molecules into MOFs at the beginning of synthesis were proposed. Here, we utilize zeolitic imidazolate frameworks (ZIFs) as an example because of their growth characteristics under mild biocompatible conditions (Zhuang et al., 2014) to describe two internally modified methods (Figure 2), which are coprecipitation (Lu et al., 2012; Shieh et al., 2015) and biomimetic mineralization (Liang et al., 2015).

The coprecipitation method was proposed by Lyu et al. (2014), who added a solution containing cytochrome c (cyt c) and polyvinylpyrrolidone (PVP) to a methanol solution of 2methylimidazole and zinc nitrate hexahydrate to achieve cytZIF nanoparticles. A transmission electron microscope (TEM) showed that $\mathrm{Zn}^{2+}$ and 2-methylimidazole were first assembled into rod-shaped crystals. Then, rhombohedral dodecahedral crystals formed $24 \mathrm{~h}$ later, indicating that ZIF-8 was formed first, before the protein was embedded. In addition, Liu demonstrated the generality of coprecipitation by loading horseradish peroxidase and lipase onto ZIF-8 and ZIF-10. The biomimetic mineralization method was inspired by a natural process according to the specific ability of amino acids, peptides, and enzymes to concentrate inorganic cations in biominerals (Trzaskowski et al., 2007; Hwang et al., 2013). This is a new and simple method to rapidly encapsulate proteins, enzymes, and DNA into MOFs, as suggested by Liang et al. (2015). Bio-ZIF could be prepared by mixing an aqueous solution containing 2-methylimidazole and bovine serum albumin (BSA) with an aqueous solution of zinc acetate at room temperature, depending on the nucleation of the ZIF-8 precursor. Coordination between the $\mathrm{Zn}$ cations and the carbonyl group of the proteins is formed naturally. Different from the crystals separated from the mixture of ZIF-8 and BSA, the BSA-ZIF synthesized by mineralization was shown to encapsulate BSA and perfectly retain the structure of the enzyme. When the biomimetically mineralized ZIF-8 layer was removed via $\mathrm{pH}$ modulation, the released biomacromolecule retained its native activity. Additionally, the category of encapsulated biomacromolecules was enlarged by biomimetic mineralization, and the crystal morphology of bioMOF had a unique dependence on the biomacromolecule.

Coprecipitation and biomimetic mineralization are generally known as "one-pot" methods because in both methods, biomolecules are initially mixed with MOF precursors and surrounded by grown MOF materials instead of occupying cavities. The difference is that PVP is utilized to functionalize the precursor and optimize the crystallization of ZIF-8 in coprecipitation. PVP is an amphiphilic, non-ionic polymer used as an efficient MOF nucleating agent to support size and shape control and stabilize nanoparticles during synthesis ( $\mathrm{Li}$ and Zhang, 2006). In the mineralization method, the nucleation of precursors in an aqueous solution allows ZIF-8 to form a protective layer without PVP. According to a comparative study of the two methods, the loading rate of protein was equivalent. Compared with that of free enzymes, biomimetic mineralization expanded the temperature range of enzymatic biological activity, that is, within a certain temperature range, biomimetic mineralization would have a better protective effect on the enzyme than coprecipitation (Liang et al., 2016a).

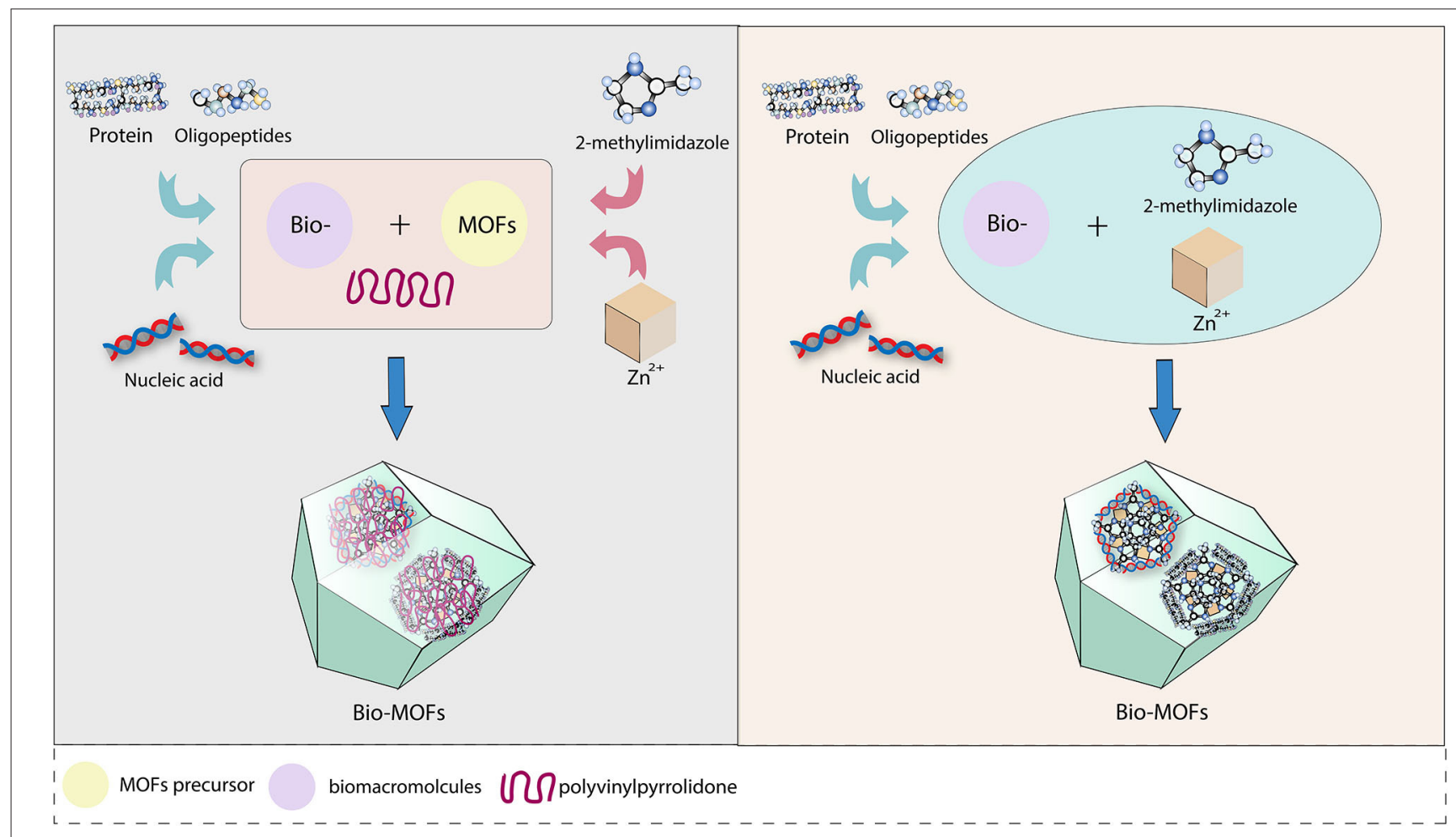

FIGURE 2 | Two methods for internal modification to MOF by biomolecule. 
In addition, the stability of the enzymes encapsulated via biomimetic mineralization was enhanced, probably due to the rigid ZIF-8 structure restricting the structural rearrangement at elevated temperatures (Hartmann and Kostrov, 2013). Moreover, the distribution of cavities throughout the crystals was analyzed by thermal enzymatic decomposition, which indicated that this was more likely to expose the enzyme to the external environment in coprecipitation. In summary, mineralization was the preferred choice for biomolecule encapsulation ( $\mathrm{He}$ et al., 2016).

In addition, the colloidal stability of the synthetic material should be considered seriously in terms of physiological factors, for example, protein-containing solution. The surface coating is proposed to stabilize the structure of nanoparticles, such as silica, (Della Rocca et al., 2011) hydrophobic polydimethysiloxane (PDMS) (Zhang et al., 2014), and hydrogel. Hydrogels are structurally various and functional materials composed of crosslinked hydrophilic polymers. The network endows a hydrogel with stability, which is a prominent advantage in embedding and protecting other materials. Additionally, the local release of cargo from a hydrogel can be achieved by degradation under specific environmental conditions. For instance, $\mathrm{Cu}-$ MOF has been embedded in poly-(polyethyleneglycol citrateco-N-isopropylacrylamide) (PPCN), which is an intrinsically antioxidant thermoresponsive citrate-based hydrogel, and MOF NPs didn't degrade in a protein solution as a result of protection by the hydrogel coating (Xiao et al., 2016). The pHsensitive carboxymethylcellulose (CMC) biopolymer was used to protect and carry the 5-FU encapsulated MOF-5 nanohybrid (5-FU@MOF-5) through the digestive system (Javanbakht et al., 2019). It should be mentioned that high biocompatibility and biodegradability are crucial properties of hydrogels, which bring numerous possibilities for use in the biomedical field ( $\mathrm{Fu}$ et al., 2018). However, electronic interactions between positively charged metal ions in MOFs and negatively charged ions in hydrogels may limit gelation. The type of metal atoms in the MOF must be considered. In a word, the hydrogel coating method can be applied to entrap MOF NPs as a mechanism to slow degradation and thus prevent aggregation.

\section{ADVANTAGES, PROSPECTS, AND CHALLENGES}

\section{Bio-MOF Advantages \\ MOFs Remarkably Protect the Activity of Biomolecules}

As nanoparticles synthesized from organic and inorganic components, MOFs are able to combine with a variety of molecules and offer superior thermal and chemical protection for their cargo (Liang et al., 2015). MOFs have been successfully developed not only in the field of drug release (Zhuang et al., 2014) but also in the study of biomacromolecules. The "micro enzyme" catalase (MP-11) and high-molecular weight molecules such as cytochrome c (104 amino acids), organophosphorus acid anhydrolase (440 amino acids) (Li et al., 2016), and bacillus subtilis lipase (BSL2) could be encapsulated by MOFs (He et al.,
2015). The framework could effectively maintain the skeletal integrity, biological activity, and reusability of proteins (Cao et al., 2016). It was reported that two enzymes, $\alpha$-glucosidase (GAA) and glucose oxidase (GOx), could be assembled on one $\mathrm{Cu}-\mathrm{MOF}$ molecule to generate a bifunctional hybrid enzymecatalytic framework reactor, which could be utilized for the highly sensitive and stable screening of GAA inhibitors (Zhong et al., 2019). In terms of nucleic acids, low-molecular weight (MW) molecules such as oligonucleotide CpG ODNs (Zhang et al., 2017) and ssDNA (11, 22, 33, and 53 nucleotides) (Peng et al., 2018) have been wrapped and combined with MOFs. However, to the best of our knowledge, it is challenging to safely deliver nucleic acids with a high MW. The latest research showed that even macromolecular plasmid DNA could be successfully embedded into a ZIF-8 carrier by the biomimetic mineralization method (Li Y. et al., 2019), so that the pDNA could be protected when it passed through the cell membrane into the lysosome and to then be released around the cell nucleus (Figure 3). Additionally, a $25-\mathrm{kD}$ polyethyleneimine (PEI) capping agent was added to improve the ZIF-8 crystal structure strength, loading capacity, $\mathrm{pH}$-responsive release, and binding affinity to pDNA. The synthetic nanoparticle pDNA@ZIF-8-PEI 25 $\mathrm{kD}$ performed better than pDNA@ZIF-8, with improved gene expression and high transfection efficacy in various types of cells, possibly because of the enhanced positive charge that facilitated binding and internalization of the nucleic acid molecule. In summary, MOFs can be used as carriers of various biomolecules and play an effective protective role during delivery.

\section{New Characteristics of MOFs Endowed by Molecules Stability}

MOF nanoparticles can be structurally affected by biomolecular modification. For example, the insertion of an enzyme was beneficial to the construction process of MOFs (Liang et al., 2016a). The binding of oligopeptides could lead to adaptive changes in metal-peptide frameworks (MPFs), which showed differences in porosity and organometallic framework strength according to the different types of amino acids used, such as glycine, threonine, and alanine (Carlos et al., 2012). In addition, lipid-functionalized MOFs have shown better stability in aqueous solution than unmodified MOFs (Wuttke et al., 2015; Zhuang et al., 2015). Liu et al. (2018) proved that Zr MOF nanoparticles bound to single-stranded DNA (ssDNA) showed better stability than did unbound particles over $24 \mathrm{~h}$. In addition, the interaction strength and pore geometry influence the absorption and release kinetics, as well as the way the matrix diffuses inside and outside the pores (Carlos et al., 2012).

\section{Targeting Movement and Local Release}

Modification not only changes the MOF structure but also endows new characteristics. Cell-targeting capabilities should be mentioned first. The mechanism of targeting is always related to the bio-MOF's bioactive group. The most common targeting method is the coupling of the ligand-receptor through distinguishing the specific recipient from the other cells. A manganese MOF modified by small cyclic arginine-glycineaspartate (RGD) peptides has been verified to target angiogenic 


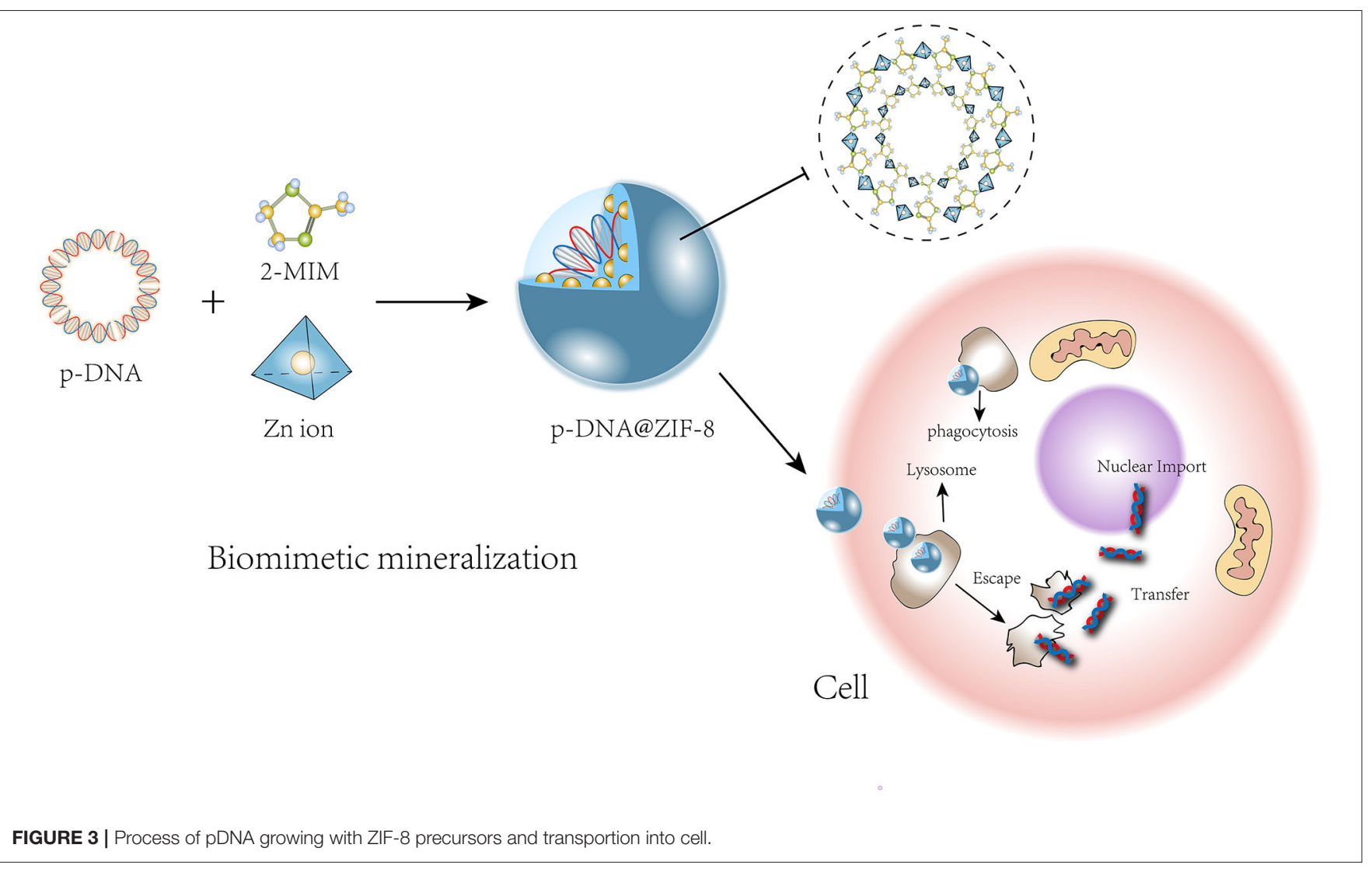

cancer cells well by binding to the upregulated $\alpha \mathrm{v} \beta 3$ integrin (Taylor et al., 2008). A terminal cyclic RGD-SH peptide was modified onto a hybrid MOF, and the nanoparticle was proven to have a targeting ability for HeLa cells (Wang D. et al., 2018). Folic acid-bovine serum albumin (FA-BSA) was a significant targeting connector for cancer cells, which resulted from efficient internalization via FA-receptors-mediated endocytosis (Jiang et al., 2018). Polysaccharide hyaluronic acid (HA) acts as a cancer-targeting ligand when connected with MOF, too, because it recognizes the overexpressed CD44 that occurs in many cancer cells (Liu et al., 2016; Kim et al., 2019). Another important mechanism of bio-MOFs' targeting movement is the sensing of chemical gradients in environments, which has previously been discovered in bacteria. We can achieve directional motion by endowing MOFs with $\mathrm{pH}$-sensitive biomolecules. Ikezoe et al. (2015) encapsulated the diphenylalanine (DPA) peptide, which could facilitate $\mathrm{Cu}-\mathrm{MOF}$ movement based on an asymmetric surface tension distribution through the dissolution and selfassembling character of DPA. The solubility of PDA is sensitive to $\mathrm{pH}$ gradients. With a higher $\mathrm{pH}$, the solubility of the DPA peptide increased, leading the MOF to lose the surface gradient, thus terminating the motion. In this way, the directional movement of MOF NPs was achieved.

In addition, controllable release is another exciting characteristic (McKinlay et al., 2010), which benefits from the fact that some kinds of MOFs can be degraded in certain environments (Park et al., 2006; Lin and Anseth, 2008). ZIF-8 has good biodegradability under acidic conditions (Della Rocca et al., 2011; Zheng et al., 2016). The ZIF-8/CpG-ODN complex showed good stability in a physiological environment, but the complex effectively released $\mathrm{CpG}$ ODNs under acidic conditions corresponding to the endolysosome identified by Toll-like receptor 9 (TLR 9). Moreover, ZIF-8 could significantly improve the uptake of CpG ODNs by RAW264.7 cells and further promote the secretion of immune cytokines in vitro and in vivo (Li Y. et al., 2019).

\section{Applications, Prospects, and Challenges}

As a novel platform for bioapplications, MOFs have made rapid progress and have constantly provided new methods for biomolecular delivery systems. MOF formation occurs because of the coordination between metal atoms and the organism, and different numbers and categories of metals or organic compounds will produce a variety of constructs, such as those resulting from bimetallic organism synthesis. Recently, a new multivariate modulation of $\mathrm{Zr}-\mathrm{MOF} \mathrm{UiO}-66$ (Abanades Lazaro et al., 2020) was reported. Multivariate modulation allows the incorporation of up to three drugs containing either carboxylates or phosphates as metal-binding units to coordinate with the defect sites of metal clusters in UiO-66. The one-pot synthesis of solvothermal compounds retained Uio-66 crystallinity and porosity so that other drugs could be further loaded. This research revealed the broader prospects and bioapplications of MOFs. MOFs are also used in the field of bioprobes. 
The $\mathrm{Cu}-\mathrm{MOF}$ was combined with a ssDNA probe labeled with carboxyfluorescein through electrostatic interactions and/or hydrogen bonding, which was used to detect $\mathrm{Hg}$ via the coordination motif between $\mathrm{Hg}$ and ssDNA (Huang et al., 2019). Another new application involved encapsulating viral nanoparticles to produce a vaccine carrier, so that the integrity of the virus and the biosafety and immunogenicity of the overall composite was enhanced due to the non-toxicity and good biocompatibility of the MOF (Luzuriaga et al., 2019).

However, the use of MOFs as potential carriers for the intracellular transmission of proteins (Liang et al., 2015) and nucleic acids (Wang S. et al., 2017; Wang Z. et al., 2017; Peng et al., 2018) is still in the preliminary stage (Alsaiari et al., 2017; Chen et al., 2018). There are some barriers that need to be resolved in the process of the bioapplication of MOFs. For example, in the future, the induction and growth-affecting factors of proteins or nucleic acids in morphological structures need further study, which will allow large double-stranded DNA molecules to be wrapped in MOFs. The biological stability of bioMOFs is another constraint. Coating may be a good choice to escape from immune system, such as poly-lactide-co-glycolide (PLGA) and cell membrane (Wang L. et al., 2018; Li J.-Y. et al., 2019). We team has invented a cell membrane-coated nanodrug deliver system to improve biocompatibility, and it has been proved the embedded drug can cleverly escape identification and clearance from the immune system, effectively prolong the blood circulation time and accurately accumulate in the target tumor tissues (Wang et al., 2020). In addition, some researchers have cautioned that most enzyme-MOF research has been focused on enzyme encapsulation in particle form, which means that solid support is essential, but this may limit the material flexibility for further practical applications (Izzah Binti Mohammad et al., 2018). Therefore, the use of versatile modalities, such as flexible ZIF-8 thin films, for the synthesis of bio-MOFs is required.

Currently, bio-MOF cascade reactions in cells have been put forward as a novel strategy. How to utilize the synergy of modified molecules in nanoparticles during working processes in organisms is still an unanswered question. For instance, in multimodal cancer therapies, the synergistic cancer starvation/ROS-mediated/chemotherapy strategy has been designed to cleverly work together in cancer cells. Glucose oxidase (GOX) modified onto the surface of $\mathrm{MOF}(\mathrm{Fe})$ catalyzes glucose into hydrogen peroxide $\left(\mathrm{H}_{2} \mathrm{O}_{2}\right)$ and gluconic acid $\left(\mathrm{H}^{+}\right)$ in cancer cells, and then $\mathrm{H}^{+}$can degrade the organic framework

\section{REFERENCES}

Abanades Lazaro, I., Wells, C. J. R., and Forgan, R. S. (2020). Multivariate modulation of the $\mathrm{Zr}$ MOF UiO-66 for defect-controlled multimodal anticancer drug delivery. Angew. Chem. Int. Ed. Engl. 132, 5249-5255. doi: 10.1002/ange.201915848

Alsaiari, S.-K., Patil, S., Alyami, M., Alamoudi, K.-O., Aleisa, F.-A., Merzaban, J.-S., et al. (2017). Endosomal escape and delivery of CRISPR/Cas9 genome editing machinery enabled by nanoscale zeolitic imidazolate framework. J. Am. Chem. Soc. 140, 143-146. doi: 10.1021/jacs.7b11754 to release camptothecin (CPT) for chemotherapy (Liu et al., 2019). In my opinion, to date, the research of bio-MOFs has always been focused on single molecules or on multiple molecules acting independently in unrelated processes. This kind of therapeutic effect is slight, but the effects will be different when we make use of all linked and correlated nodes in entire reaction chains, such as the chain of ROS-induced oxidation. In the latest research, $\mathrm{MOF}(\mathrm{Fe})$ is used to promote ROS-induced oxidative damage in cancer cells, and chloroquine modification will inhibit lysosome autophagy, so that this nanodrug can cut off the self-protection node under the oxidative stress chain and improve the anti-cancer effect (Yang et al., 2020). As we can see, the regulation of multiple reactive nodes in a biological chain can be achieved by setting the reaction times of substances loaded onto MOFs. I believe that achieving synergistic effects to maximize the efficiency of bio-MOFs will be a point that attracts the focus of scientists.

Finally, the development direction of bio-MOFs must be toward clinical applications, and transformations are in development, such as targeted protein or DNA biosensors (Osman et al., 2019) and delivery (Wang S. et al., 2019). There is a wider range of utilization of bio-MOFs. Through interdisciplinary research, bio-MOFs will have a significant impact on various fields including chemistry, genetics, biology, and materials science (Wu et al., 2018). We hope that genetic diagnosis, biological-targeted therapy, and therapeutic drug encapsulation can be improved through the use of MOFs in the future.

\section{AUTHOR CONTRIBUTIONS}

QX, YH, and LW contributed conception and design of the study. QX wrote the first draft of the manuscript. LW revised the manuscript. YP wrote sections of the manuscript. All authors contributed to manuscript revision, read, and approved the submitted version.

\section{FUNDING}

We gratefully appreciate the financial support from the National Natural Science Foundation of China (81601883 and 81873988 ) and the Science and Technology Innovation Project sponsored by the China Hunan Provincial Science \& Technology Department (2017SK50106). 
Cao, Y., Wu, Z., Wang, T., Xiao, Y., Huo, Q., and Liu, Y. (2016). Immobilization of Bacillus subtilis lipase on a Cu-BTC based hierarchically porous metalorganic framework material: a biocatalyst for esterification. Dalton Trans. 45, 6998-7003. doi: 10.1039/C6DT00677A

Carlos, M.-G., Warren, J.-E., Stylianou, K.-C., Flack, N.-L., and Rosseinsky M.J. (2012). Enhanced stability in rigid peptide-based porous materials. Angew. Chem. Int. Ed. Engl. 51, 11044-11048. doi: 10.1002/anie.201203929

Chen, T.-T., Yi, J.-T., Zhao, Y.-Y., and Chu, X. (2018). Biomineralized metalorganic framework nanoparticles enable intracellular delivery and endolysosomal release of native active proteins. J. Am. Chem. Soc. 140, 9912-9920. doi: 10.1021/jacs.8b04457

Chen, Y., Lykourinou, V., Vetromile, C., Hoang, T., Ming, L.-J., and Larsen, R. W. (2012). How can proteins enter the interior of a MOF? Investigation of cytochrome $c$ translocation into a MOF consisting of mesoporous cages with microporous windows. J. Am. Chem. Soc. 134, 13188-13191. doi: $10.1021 /$ ja305144x

Cheng, X., and Lee, R.-J. (2016). The role of helper lipids in lipid nanoparticles (LNPs) designed for oligonucleotide delivery. Adv. Drug Delivery Rev. 99, 129-137. doi: 10.1016/j.addr.2016.01.022

Chira, S., Jackson, C.-S., Oprea, I., Ozturk, F., Pepper, M.-S., Diaconu, I., et al. (2015). Progresses towards safe and efficient gene therapy vectors. Oncotarget 6:30675. doi: 10.18632/oncotarget.5169

Chowdhuri, A.-R., Das, B., Kumar, A., Tripathy, S., Roy, S., and Sahu, S.-K. (2017). One-pot synthesis of multifunctional nanoscale metal-organic frameworks as an effective antibacterial agent against multidrug-resistantStaphylococcus aureus. Nanotechnology 28:095102. doi: 10.1088/1361-6528/aa57af

Das, S. K., Menezes, M. E., Bhatia, S., Wang, X. Y., Emdad, L., and Sarkar, D. (2015). Gene therapies for cancer: strategies, challenges and successes. J. Cell. Physiol. 230, 259-271. doi: 10.1002/jcp.24791

Dash, P.-R., Read, M.-L., Barrett, L.-B., Wolfert, M.-A., and Seymour, L.-W. (1999). Factors affecting blood clearance and in vivo distribution of polyelectrolyte complexes for gene delivery. Gene Ther. 6, 643-650. doi: 10.1038/sj.gt.3300843

Della Rocca, J., Liu, D., and Lin, W. (2011). Nanoscale metal-organic frameworks for biomedical imaging and drug delivery. Acc. Chem. Res. 44, 957-968. doi: $10.1021 / \operatorname{ar} 200028$ a

Deria, P., Bury, W., Hod, I., Kung, C.-W., Karagiaridi, O., Hupp, J. T., et al. (2015). MOF functionalization via solvent-assisted ligand incorporation: phosphonates vs carboxylates. Inorg. Chem. 54, 2185-2192. doi: 10.1021/ic502639v

Doonan, C., Riccò, R., Liang, K., Bradshaw, D., and Falcaro, P. (2017). Metalorganic frameworks at the biointerface: synthetic strategies and applications. Acc. Chem. Res. 50, 1423-1432. doi: 10.1021/acs.accounts.7b00090

Eddaoudi, M., Kim, J., Rosi, N., Vodak, D., Wachter, J., O’Keeffe, M., et al. (2002). Systematic design of pore size and functionality in isoreticular MOFs and their application in methane storage. Science 295, 469-472. doi: $10.1126 /$ science. 1067208

Férey, G. (2008). Hybrid porous solids: past, present, future. Chem. Soc. Rev. 37, 191-214. doi: 10.1039/B618320B

Fu, L.-H., Qi, C., Ma, M.-G., and Wan, P. (2018). Multifunctional cellulosebased hydrogels for biomedical applications. J. Mater. Chem. B 7, 1541-1562. doi: $10.1039 / \mathrm{c} 8$ tb02331j

Ginn, S.-L., Amaya, A. K., Alexander, I. E., Edelstein, M., and Abedi, M. R. (2018). Gene therapy clinical trials worldwide to 2017: an update. J. Gene Med. 20:e3015. doi: 10.1002/jgm.3015

Hartmann, M., and Kostrov, X. (2013). Immobilization of enzymes on porous silicas-benefits and challenges. Chem. Soc. Rev. 42:6277. doi: $10.1039 / \mathrm{c} 3 \operatorname{cs} 60021 \mathrm{a}$

He, C., Liu, D., and Lin, W. (2015). Nanomedicine applications of hybrid nanomaterials built from metal-ligand coordination bonds: nanoscale metalorganic frameworks and nanoscale coordination polymers. Chem. Rev. 115, 11079-11108. doi: 10.1021/acs.chemrev.5b00125

He, H., Han, H., Shi, H., Tian, Y., Sun, F., and Song, Y. (2016). Construction of thermophilic lipase-embedded metal-organic frameworks via biomimetic mineralization: a biocatalyst for ester hydrolysis and kinetic resolution. ACS Appl. Mater. Interfaces 8, 24517-24524. doi: 10.1021/acsami.6b05538

Horcajada, P., Chalati, T., Serre, C., Gillet, B., Sebrie, C., Baati, T., et al. (2009). Porous metal-organic-framework nanoscale carriers as a potential platform for drug delivery and imaging. Nat. Mater. 9, 172-178. doi: 10.1038/nmat2608
Horcajada, P., Gref, R., Baati, T., Allan, P.-K., Maurin, G., Couvreur, P., et al. (2011). Metal-organic frameworks in biomedicine. Chem. Rev. 112, 1232-1268. doi: $10.1021 / \mathrm{cr} 200256 \mathrm{v}$

Huang, N.-H., Li, R.-T., Fan, C., Wu, K.-Y., Zhang, Z., and Chen, J.X. (2019). Rapid sequential detection of $\mathrm{Hg}^{2+}$ and biothiols by a probe DNA-MOF hybrid sensory system. J. Inorg. Biochem. 197:110690. doi: 10.1016/j.jinorgbio.2019.04.004

Huang, X., He, Z., Guo, D., Liu, Y., Song, J., Yung, B. C., et al. (2018). "Three-in-one" nanohybrids as synergistic nanoquenchers to enhance nowash fluorescence biosensors for ratiometric detection of cancer biomarkers. Theranostics 8, 3461-3473. doi: 10.7150/thno.25179

Hudson, S., Cooney, J., and Magner, E. (2008). Proteins in mesoporous silicates. Angew. Chem. Int. Ed. Engl. 47, 8582-8594. doi: 10.1002/anie.200705238

Huxford, R. C., Della Rocca, J., and Lin, W. (2010). Metal-organic frameworks as potential drug carriers. Curr. Opin. Chem. Biol. 14, 262-268. doi: 10.1016/j.cbpa.2009.12.012

Hwang, E.-T., Tatavarty, R., Chung, J., and Gu, M.-B. (2013). New functional amorphous calcium phosphate nanocomposites by enzymeassisted biomineralization. ACS Appl. Mater. Interfaces 5, 532-537. doi: 10.1021/am302580p

Ikezoe, Y., Fang, J., Wasik, T.-L., Shi, M., Uemura, T., Kitagawa, S., et al. (2015). Peptide-metal organic framework swimmers that direct the motion toward chemical targets. Nano Lett. 15, 4019-4023. doi: 10.1021/acs.nanolett.5b00969

Imaz, I., Rubio-Martínez, M., An, J., Solé-Font, I., Rosi, N. L., and Maspoch, D. (2011). Metal-biomolecule frameworks (MBioFs). Chem. Commun. 47, 7287-7302. doi: 10.1039/c1cc11202c

Izzah Binti Mohammad, M., Razmjou, A., Liang, K., Asadnia, M., and Chen, V. (2018). MOF-based enzymatic microfluidic biosensor via surface patterning and biomineralization. ACS Appl. Mater. Interfaces 11, 1807-1820. doi: 10.1021/acsami.8b16837

Javanbakht, S., Hemmati, A., Namazi, H., and Heydari, A. (2019). Carboxymethylcellulose-coated 5-fluorouracil@MOF-5 nano-hybrid as a bio-nanocomposite carrier for the anticancer oral delivery. Int. J. Biol. Macromol. 155, 876-882. doi: 10.1016/j.ijbiomac.2019.12.007

Jiang, W., Zhang, H., Wu, J., Zhai, G., Li, Z., and Luan, Y. (2018).CuS@MOF-based well-designed quercetin delivery system for chemo-photothermal therapy. ACS Appl. Mater. Interfaces 10, 34513-34523. doi: 10.1021/acsami.8b13487

Keles, E., Song, Y., Du, D., Dong, W.-J., and Lin, Y. (2016). Recent progress in nanomaterials for gene delivery applications. Biomater. Sci. 4, 1291-1309. doi: 10.1039/C6BM00441E

Khalil, I. A., Kogure, K., Akita, H., and Harashima, H. (2006). Uptake pathways and subsequent intracellular trafficking in nonviral gene delivery. Pharmacol. Rev. 58, 32-45. doi: 10.1124/pr.58.1.8

Kim, K., Lee, S., Jin, E., Palanikumar, L., Lee, J.-H., Kim, J.-C., et al. (2019). MOF X biopolymer: collaborative combination of metal-organic framework and biopolymer for advanced anticancer therapy. ACS Appl. Mater. Interfaces 11, 27512-27520. doi: 10.1021/acsami.9b05736

Kotterman, M.-A., Chalberg, T.-W., and Schaffer, D.-V. (2015). Viral vectors for gene therapy: translational and clinical outlook. Annu. Rev. Biomed. Eng. 17, 63-89. doi: 10.1146/annurev-bioeng-071813-104938

Li, J.-Y., Hu, H.-Y., Jiang, Z.-C., Chen, S.-J., Pan, Y.-X., Guo, Q., et al. (2019). Near-infrared-induced IR780-loaded PLGA nanoparticles for photothermal therapy to treat breast cancer metastasis in bones. RSC Adv. 9, 35976-35983. doi: 10.1039/C9RA05813C

Li, J. R., Kuppler, R. J., and Zhou, H. C. (2009). Selective gas adsorption and separation in metal-organic frameworks. Chem. Soc. Rev. 38, 1477-1504. doi: 10.1039/b802426j

Li, P., Moon, S.-Y., Guelta, M.-A., Harvey, S.-P., Hupp, J.-T., and Farha, O.-K. (2016). Encapsulation of a nerve agent detoxifying enzyme by a mesoporous zirconium metal-organic framework engenders thermal and longterm stability. J. Am. Chem. Soc. 138, 8052-8055. doi: 10.1021/jacs.6b03673

Li, Y., Zhang, K., Liu, P., Chen, M., Zhong, Y., Ye, Q., et al. (2019). Encapsulation of plasmid dna by nanoscale metal-organic frameworks for efficient gene transportation and expression. Adv. Mater. 31:1901570. doi: 10.1002/adma.201901570

Li, Z., and Zhang, Y. (2006). Monodisperse silica-coated polyvinylpyrrolidone/NaYF4 nanocrystals with multicolor upconversion 
fluorescence emission. Angew. Chem. Int. Ed. Engl. 45, 7732-7735. doi: $10.1002 /$ anie. 200602975

Liang, K., Coghlan, C.-J., Bell, S.-G., Doonan, C., and Falcaro, P. (2016a). Enzyme encapsulation in zeolitic imidazolate frameworks: a comparison between controlled co-precipitation and biomimetic mineralisation. Chem. Commun. 52, 473-476. doi: 10.1039/C5CC07577G

Liang, K., Ricco, R., Doherty, C.-M., Styles, M.-J., Bell, S., Kirby, N., et al. (2015). Biomimetic mineralization of metal-organic frameworks as protective coatings for biomacromolecules. Nat. Commun. 6:7240. doi: 10.1038/ncomms8240

Liang, K., Richardson, J.-J., Cui, J., Caruso, F., Doonan, C.-J., and Falcaro, P. (2016b). Metal-organic framework coatings as cytoprotective exoskeletons for living cells. Adv. Mater. 28, 7910-7914. doi: 10.1002/adma.201602335

Lin, C.-C., and Anseth, K.-S. (2008). PEG hydrogels for the controlled release of biomolecules in regenerative medicine. Pharm. Res. 26, 631-643. doi: 10.1007/s11095-008-9801-2

Liu, C., Chen, Z., Wang, Z., Li, W., Ju, E., Yan, Z., et al. (2016). A graphitic hollow carbon nitride nanosphere as a novel photochemical internalization agent for targeted and stimuli-responsive cancer therapy. Nanoscale 8, 12570-12578. doi: 10.1039/C5NR07719B

Liu, Y., Hou, W., Xia, L., Cui, C., Wan, S., Jiang, Y., et al. (2018). ZrMOF nanoparticles as quenchers to conjugate DNA aptamers for targetinduced bioimaging and photodynamic therapy. Chem. Sci. 9, 7505-7509. doi: $10.1039 / \mathrm{C} 8 \mathrm{SC} 02210 \mathrm{~K}$

Liu, Z., Li, T., Han, F., Gan, Y., Wang, Y., Shi, J., et al. (2019). A cascade-reaction enabled synergistic cancer starvation/ROS-mediated/chemotherapy with enzyme modified Fe-based MOF. Biomater. Sci. 7, 3683-3692. doi: 10.1039/C9BM00641A

Lu, G., Li, S., Guo, Z., Farha, O.-K., Hauser, B.-G., Qi, X., et al. (2012). Imparting functionality to a metal-organic framework material by controlled nanoparticle encapsulation. Nat. Chem. 4, 310-316. doi: 10.1038/nchem.1272

Luzuriaga, M.-A., Welch, R.-P., Dharmarwardana, M., Benjamin, C.-E., Li, S., Shahrivarkevishahi, A., et al. (2019). Enhanced stability and controlled delivery of MOF encapsulated vaccines and their immunogenic response in vivo. ACS Appl. Mater. Interfaces 11, 9740-9746. doi: 10.1021/acsami.8b20504

Lv, H., Zhang, S., Wang, B., Cui, S., and Yan, J. (2006). Toxicity of cationic lipids and cationic polymers in gene delivery. J. Control. Release 114, 100-109. doi: 10.1016/j.jconrel.2006.04.014

Lykourinou, V., Chen, Y., Wang, X.-S., Meng, L., Hoang, T., Ming, L.-J., et al. (2011). Immobilization of MP-11 into a mesoporous metal-organic framework, MP-11@mesoMOF: a new platform for enzymatic catalysis. J. Am. Chem. Soc. 133, 10382-10385. doi: 10.1021/ja2038003

Lyu, F., Zhang, Y., Zare, R.-N., Ge, J., and Liu, Z. (2014). One-pot synthesis of protein-embedded metal-organic frameworks with enhanced biological activities. Nano Lett. 14, 5761-5765. doi: 10.1021/nl5026419

Mantion, A., Massüger, L., Rabu, P., Palivan, C., McCusker, L. B., and Taubert, A. (2008). Metal-peptide frameworks (MPFs): "bioinspired" metal organic frameworks. J. Am. Chem. Soc. 130, 2517-2526. doi: 10.1021/ja0762588

McKinlay, A. C., Morris, R. E., Horcajada, P., Férey, G., Gref, R., Couvreur, P., et al. (2010). BioMOFs: metal-organic frameworks for biological and medical applications. Angew. Chem. Int. Ed. Engl. 49, 6260-6266. doi: $10.1002 /$ anie. 201000048

Meng, H.-M., Hu, X.-X., Kong, G.-Z., Yang, C., Fu, T., Li, Z.-H., et al. (2018). Aptamer-functionalized nanoscale metal-organic frameworks for targeted photodynamic therapy. Theranostics 8, 4332-4344. doi: 10.7150/thno.26768

Mintzer, M.-A., and Simanek, E.-E. (2009). Nonviral vectors for gene delivery. Chem. Rev. 109, 259-302. doi: 10.1021/cr800409e

Morris, R. E., and Wheatley, P. S. (2008). Gas storage in nanoporous materials. Angew. Chem. Int. Ed. Engl. 47, 4966-4981. doi: 10.1002/anie.200703934

Morris, W., Briley, W.-E., Auyeung, E., Cabezas, M. D., and Mirkin, C.-A. (2014). Nucleic acid-metal organic framework (MOF) nanoparticle conjugates. J. Am. Chem. Soc. 136, 7261-7264. doi: 10.1021/ja503215w

Nam, H.-Y., Park, J.-H., Kim, K., Kwon, I.-C., and Jeong, S.-Y. (2009). Lipid-based emulsion system as non-viral gene carriers. Arch. Pharm. Res. 32, 639-646. doi: 10.1007/s12272-009-1500-y

Osman, D. I., El-Sheikh, S. M., Sheta, S. M., Ali, O. I., Salem, A. M., Shousha, W. G., et al. (2019). Nucleic acids biosensors based on metal-organic framework (MOF): paving the way to clinical laboratory diagnosis. Biosens. Bioelectron. 141:111451. doi: 10.1016/j.bios.2019.111451
Park, K.-S., Ni, Z., Cote, A.-P., Choi, J.-Y., Huang, R., and Uribe-Romo, F.-J. (2006). Exceptional chemical and thermal stability of zeolitic imidazolate frameworks. Proc. Natl. Acad. Sci. U.S.A. 103, 10186-10191. doi: $10.1073 /$ pnas.0602439103

Peng, S., Bie, B., Sun, Y., Liu, M., Cong, H., Zhou, W., et al. (2018). Metal-organic frameworks for precise inclusion of single-stranded DNA and transfection in immune cells. Nat. Commun. 9:1293. doi: 10.1038/s41467-018-03650-w

Rapoport, T.-A. (2007). Protein translocation across the eukaryotic endoplasmic reticulum and bacterial plasma membranes. Nature 450, 663-669. doi: 10.1038/nature06384

Roth, S.-K., Epley, C.-C., Novak, J.-J., McAndrew, M.-L., Cornell, H.-D., Zhu J., et al. (2018). Photo-triggered release of 5-fluorouracil from a MOF drug delivery vehicle. Chem. Commun. 54, 7617-7620. doi: 10.1039/C8CC01601A

Shekhah, O., Liu, J., Fischer, R.-A., and Wöll, C. (2011). MOF thin films: existing and future applications. Chem. Soc. Rev. 40:1081. doi: 10.1039/c0cs00147c

Shieh, F.-K., Wang, S.-C., Yen, C.-I., Wu, C.-C., Dutta, S., Chou, L.-Y., et al. (2015). Imparting functionality to biocatalysts via embedding enzymes into nanoporous materials by a de novo approach: size-selective sheltering of catalase in metal-organic framework microcrystals. J. Am. Chem. Soc. 137, 4276-4279. doi: 10.1021/ja513058h

Sokolova, V., and Epple, M. (2008). Inorganic nanoparticles as carriers of nucleic acids into cells. Angew. Chem. Int. Ed. Engl. 47, 1382-1395. doi: $10.1002 /$ anie. 200703039

Taylor, K.-M., Rieter, W.-J., and Lin, W. (2008). Manganese-based nanoscale metal-organic frameworks for magnetic resonance imaging. J. Am. Chem. Soc. 130, 14358-14359. doi: 10.1021/ja803777x

Thomas, C.-E., Ehrhardt, A., and Kay, M.-A. (2003). Progress and problems with the use of viral vectors for gene therapy. Nat. Rev. Genet. 4, 346-358. doi: $10.1038 / \operatorname{nrg} 1066$

Trzaskowski, B., Adamowicz, L., and Deymier, P.-A. (2007). A theoretical study of zinc(II) interactions with amino acid models and peptide fragments. J. Biol. Inorg. Chem. 13, 133-137. doi: 10.1007/s00775-007-0306-y

Tseng, Y. C., Mozumdar, S., and Huang, L. (2009). Lipid-based systemic delivery of siRNA. Adv. Drug Deliv. Rev. 61, 721-731. doi: 10.1016/j.addr.2009.03.003

Waehler, R., Russell, S.-J., and Curiel, D.-T. (2007). Engineering targeted viral vectors for gene therapy. Nat. Rev. Genet. 8, 573-587. doi: 10.1038/nrg2141

Wan, C., Allen, T.-M., and Cullis, P.-R. (2013). Lipid nanoparticle delivery systems for siRNA-based therapeutics. Drug Deliv. Transl. Res. 4, 74-83. doi: 10.1007/s13346-013-0161-z

Wang, D., Wu, H., Zhou, J., Xu, P., Wang, C., Shi, R., et al. (2018). In situ one-pot synthesis of MOF-polydopamine hybrid nanogels with enhanced photothermal effect for targeted cancer therapy. Adv. Sci. 5:1800287. doi: 10.1002 /advs. 201800287

Wang, D., Zhao, C., Gao, G., Xu, L., Wang, G., and Zhu, P. (2019). Multifunctional $\mathrm{NaLnF}_{4} @ \mathrm{MOF}-\mathrm{Ln}$ nanocomposites with dual-mode luminescence for drug delivery and cell imaging. Nanomaterials 9:1274. doi: 10.3390/nano9091274

Wang, L., Chen, S., Pei, W., Huang, B., and Niu, C. (2020). Magnetically targeted erythrocyte membrane coated nanosystem for synergistic photothermal/chemotherapy of cancer. J. Mater. Chem. B 8, 4132-4142. doi: 10.1039/D0TB00364F

Wang, L., Chen, S., Zhu, Y., Zhang, M., Tang, S., Li, J., et al. (2018). Triple-modal imaging-guided chemo-photothermal synergistic therapy for breast cancer with magnetically targeted phase-shifted nanoparticles. ACS Appl. Mater. Interfaces 10, 42102-42114. doi: 10.1021/acsami.8b16323

Wang, S., Chen, Y., Wang, S., Li, P., Mirkin, C. A., and Farha, O. K. (2019). DNAfunctionalized metal-organic framework nanoparticles for intracellular delivery of proteins. J. Am. Chem. Soc. 141, 2215-2219. doi: 10.1021/jacs.8b12705

Wang, S., McGuirk, C.-M., Ross, M.-B., Wang, S., Chen, P., Xing, H., et al. (2017). General and direct method for preparing oligonucleotide-functionalized metal-organic framework nanoparticles. J. Am. Chem. Soc. 139, 9827-9830. doi: $10.1021 /$ jacs.7b05633

Wang, S., Morris, W., Liu, Y., McGuirk, C.-M., Zhou, Y., Hupp, J.-T., et al. (2015). Surface-specific functionalization of nanoscale metal-organic frameworks. Angew. Chem. Int. Ed. Engl. 54, 14738-14742. doi: 10.1002/anie.201506888

Wang, Z., Fu, Y., Kang, Z., Liu, X., Chen, N., Wang, Q., et al. (2017). Organelle-specific triggered release of immunostimulatory oligonucleotides from intrinsically coordinated DNA-metal-organic frameworks with soluble exoskeleton. J. Am. Chem. Soc. 139, 15784-15791. doi: 10.1021/jacs.7b07895 
Whitehead, K.-A., Langer, R., and Anderson, D.-G. (2009). Knocking down barriers: advances in siRNA delivery. Nat. Rev. Drug Discovery 8, 129-138. doi: $10.1038 / \mathrm{nrd} 2742$

Wickner, W. (2005). Protein translocation across biological membranes. Science 310, 1452-1456. doi: 10.1126/science. 1113752

Wu, Q., Niu, M., Chen, X., Tan, L., Fu, C., Ren, X., et al. (2018). Biocompatible and biodegradable zeolitic imidazolate framework/polydopamine nanocarriers for dual stimulus triggered tumor thermo-chemotherapy. Biomaterials 162, 132-143. doi: 10.1016/j.biomaterials.2018.02.022

Wuttke, S., Braig, S., Preiß, T., Zimpel, A., Sicklinger, J., Bellomo, C., et al. (2015). MOF nanoparticles coated by lipid bilayers and their uptake by cancer cells. Chem. Commun. 51, 15752-15755. doi: 10.1039/C5CC06767G

Wyszogrodzka-Gaweł, G., Dorozynski, P., Giovagnoli, S., Strzempek, W., Pesta, E., Weglarz, W. P., et al. (2019). An inhalable theranostic system for local tuberculosis treatment containing an isoniazid loaded metal organic framework Fe-MIL-101-NH2-from raw MOF to drug delivery system. Pharmaceutics 11:687. doi: 10.3390/pharmaceutics11120687

Xiao, B., Byrne, P.-J., Wheatley, P.-S., Wragg, D.-S., Zhao, X., and Fletcher, A.J. (2009). Chemically blockable transformation and ultraselective low-pressure gas adsorption in a non-porous metal organic framework. Nat. Chem. 1, 289-294. doi: 10.1038/nchem.254

Xiao, J., Chen, S., Yi, J., Zhang, H.-F., and Ameer, G. A. (2016). A cooperative copper metal-organic framework-hydrogel system improves wound healing in diabetes. Adv. Funct. Mater. 27:1604872. doi: 10.1002/adfm.201604872

Xu, H., Liu, F., Cui, Y., Chen, B., and Qian, G. (2011). A luminescent nanoscale metal-organic framework for sensing of nitroaromatic explosives. Chem. Commun. 47:3153. doi: 10.1039/c0cc05166g

Yang, B., Ding, L., Yao, H., Chen, Y., and Shi, J. (2020). A metalorganic framework (MOF) Fenton nanoagent-enabled nanocatalytic cancer therapy in synergy with autophagy inhibition. Adv. Mater. 32:1907152. doi: 10.1002/adma.201907152

Yin, H., Kanasty, R. L., Eltoukhy, A. A., Vegas, A. J., Dorkin, J. R., and Anderson, D. G. (2014). Non-viral vectors for gene-based therapy. Nat. Rev. Genet. 15, 541-555 doi: 10.1038/nrg3763

Yoo, J. W., Irvine, D. J., Discher, D. E., and Mitragotri, S. (2011). Bio-inspired, bioengineered and biomimetic drug delivery carriers. Nat. Rev. Drug. Discovery 10, 521-535. doi: 10.1038/nrd3499

Zhang, H., Chen, W., Gong, K., and Chen, J. (2017). Nanoscale zeolitic imidazolate framework-8 as efficient vehicles for enhanced delivery of CpG oligodeoxynucleotides. ACS Appl. Mater. Interfaces 9, 31519-31525. doi: 10.1021/acsami.7b09583
Zhang, W., Hu, Y., Ge, J., Jiang, H.-L., and Yu, S.-H. (2014). A facile and general coating approach to moisture/water-resistant metal-organic frameworks with intact porosity. J. Am. Chem. Soc. 136, 16978-16981. doi: 10.1021/ja5 09960n

Zhao, D., Tan, S., Yuan, D., Lu, W., Rezenom, Y.-H., et al. (2011). Surface functionalization of porous coordination nanocages via click chemistry and their application in drug delivery. Adv. Mater. 23, 90-93. doi: 10.1002/adma.201003012

Zheng, H., Zhang, Y., Liu, L., Wan, W., Guo, P., Nyström, A. M., et al. (2016). Onepot synthesis of metal-organic frameworks with encapsulated target molecules and their applications for controlled drug delivery. J. Am. Chem. Soc. 138, 962-968. doi: 10.1021/jacs.5b11720

Zhong, Y., Yu, L., He, Q., Zhu, Q., Zhang, C., Cui, X., et al. (2019). Bifunctional hybrid enzyme-catalytic metal organic framework reactor for $\alpha$-glucosidase inhibitor screening. ACS Appl. Mater. Interfaces 11:32769. doi: 10.1021/acsami.9b11754

Zhou, H.-C., Long, J.-R., and Yaghi, O.-M. (2012). Introduction to metal organic frameworks. Chem. Rev. 112, 673-674. doi: 10.1021/cr3 $00014 x$

Zhuang, J., Chou, L.-Y., Sneed, B.-T., Cao, Y., Hu, P., and Feng, L., et al. (2015). Surfactant-mediated conformal overgrowth of core-shell metal-organic framework materials with mismatched topologies. Small 11, 5551-5555. doi: $10.1002 /$ smll.201501710

Zhuang, J., Kuo, C.-H., Chou, L.-Y., Liu, D.-Y., Weerapana, E., and Tsung, C.-K. (2014). Optimized metal-organic-framework nanospheres for drug delivery: evaluation of small-molecule encapsulation. ACS Nano 8:2812-2819. doi: $10.1021 / \mathrm{nn} 406590 \mathrm{q}$

Zhuang, J., Young, A.-P., and Tsung, C.-K. (2017). Integration of biomolecules with metal-organic frameworks. Small 13:1700880. doi: $10.1002 / \mathrm{smll} .201700880$

Conflict of Interest: The authors declare that the research was conducted in the absence of any commercial or financial relationships that could be construed as a potential conflict of interest.

Copyright (C) 2020 Xing, Pan, Hu and Wang. This is an open-access article distributed under the terms of the Creative Commons Attribution License (CC BY). The use, distribution or reproduction in other forums is permitted, provided the original author(s) and the copyright owner(s) are credited and that the original publication in this journal is cited, in accordance with accepted academic practice. No use, distribution or reproduction is permitted which does not comply with these terms. 